\section{Hormone balance}

\section{S.J. Simpson}

Endocrinology of Insects. Invertebrate

Endocrinology, Vol.1.

Edited by Roger G.H. Downer and Hans Laufer.

Alan R. Liss/Wiley: 1984. Pp.707.

$\$ 199.80$, f1I1.

ONE of the most daunting tasks in biology would be to prepare a volume entitled Endocrinology of Insects. So daunting, in fact, that until now no one has attempted to do more than review selected aspects of what is an enormous, complex and rapidly expanding field. Downer and Laufer have approached the problem of producing a detailed, scholarly review of the subject in exactly the right, and probably the only way: they have asked specialists to cover defined ground.

The resulting volume deals with the nature of endocrine glands and neurosecretory cells; the chemistry and biosynthesis of hormones, and their interaction with target cells; intracellular second-messengers; the physiological role of hormones; myotropic

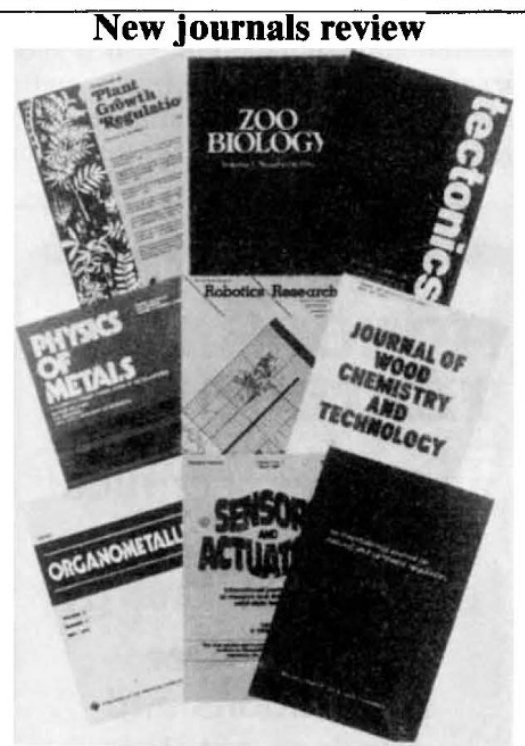

On 27 September Nature will publish the fourth annual review supplement devoted to science journals.

Criteria for inclusion of a journal in the 1984 issue are that:

(i) the first number appeared, or the journal was retitled, between June 1982 and May 1983 (the second cut-off date allows at least three issues of a journal to have been published, the minimum number on which a reasonable judgement can be based);

(ii) it is published at least three times a year;

(iii) the main language used is English.

Publishers and learned societies are invited to send four different issues of each suitable periodical, including the first and most recent numbers (if from outside the United Kingdom, by air mail) to: The Review Editor, Nature, 4 Little Essex Street, London WC2R 3LF, England. Subscription details, if possible for both 1984 and 1985, should be included. and neurotropic substances; pheromones; the relevance of insect hormones in plants; and the usefulness of hormones in insect control. Inevitably there are omissions, and problems of redundancy and lack of continuity, but the volume as a whole is a success. The differing interpretations and standpoints expressed by the contributors do, as the editors claim, provide depth and balance to the work.

Endocrinology of Insects is not easy to read; not, in most cases, because the writing is unclear, but because the authors have done justice to their topics. This approach has often precluded the drawing of generalizations or syntheses. Even the introductory overviews for such sections as "Regulation of Metamorphosis", "Regulation of [Female] Reproduction"' (unfortunately male reproduction is not represented) and "Metabolic Homeostasis" are, by necessity, unable to do more than draw together information in a superficial sense. In most cases, not enough is known to generalize, and what is known is often conflicting different species use the same hormone for different purposes; different strains of the same species, and even a single strain under different rearing regimes, give conflicting results; it is difficult to distinguish real effects from those stemming from dissection and bioassay techniques; and there is a danger that phenomena are attributed to a particular hormone when in fact the hormone is acting at an earlier stage in a causal sequence involving other endocrine and neural factors. More aspects of insect endocrinology will, perhaps, soon begin to gel, but the cautious approach of many of the contributors is generally justified.

An unusual aspect of the book is the coverage of areas about which virtually nothing is known. Thus the section on metabolic homeostasis includes three detailed chapters on excretion, on lipid metabolism and on carbohydrate metabolism, along with a chapter on nitrogen metabolism in which the word hormone is almost absent. Such a contrast emphasizes those areas which are poorly understood and puts the better known subjects in perspective. The quest for completeness has its drawbacks, however. For example, neurotransmitters, neuromodulators and pheromones differ from hormones in degree rather than kind and the editors have chosen to represent them but briefly - in the case of pheromones, too briefly to be of much use.

I would have liked more thorough crossreferencing between chapters, but that apart my main worry is that the book is two years out of date. Nonetheless, Endocrinology of Insects is a very important work. An understanding of hormonal mechanisms is essential to anyone who studies insect behaviour or physiology, and this book makes a difficult subject accessible to us all.

S.J. Simpson is Demonstrator in the Depart ment of Zoology, University of Oxford.
Screening methods of recombination

\section{E.J. Wood}

The Techniques in Genetic Engineering Video Library. TGE 1: Nucleic Acids

Techniques - An Overview.

By Stephen D.M. Brown.

IRL Press: 1984. Formats available: PAL in VHS and Betamax, $£ 80, \$ 160$. U-matic copies $£ 7.50, \$ 15$ extra. Supplementary charge for NTSC and SECAM standards conversions.

THIS 25-minute videotape is the first of a series of eight* which will deal with the techniques of genetic engineering. Viewed in isolation from the rest of the programmes (which will appear later this year), it gives a foretaste of the high quality of production of the series, but tells us less about what can be expected of the content and level of the subsequent tapes. Nor is it yet clear how easily a student could proceed from instruction of this type to actual work in the laboratory.

The programme starts with a graphic display of a computer-generated DNA double helix, the introductory section then featuring a series of stills during which the voiceover talks about the impact of genetic engineering and its potential and limitations. A brief interview with Sir Gordon Wolstenholme gives an historical view of developments since Watson and Crick, and stresses that no one really saw any practical uses for the techniques of genetic manipulation until seven or eight years ago.

Following on, the fundamental techniques underlying recombinant DNA technology are explained, namely the use of restriction enzymes, of sticky ends and of ligases. The use of restriction enzymes is well illustrated with graphics, although I wonder if it is really necessary at this level to speak about the different classes of restriction enzyme. Analysis by restriction mapping is demonstrated, and from this a student would readily get a good feel for how slabs of agar are used in electrophoresis, blotting and so on. On the whole this section is evenly paced, well-explained and clearly photographed.

The next section deals with the cloning of DNA into a phage in order ". . . to look at a single gene". Graphics show the ligation of a section of "human" DNA into phage DNA, reinforcing what had been explained in the previous section. However at this stage the graphics seem to get out of phase with the voice-over: while the latter talks about plaques in a lawn of bacteria, the graphics show thousands of virus particles.

* Titles to follow are Gene Analysis and Southern Blotting; DNA Sequencing with MI3; Gene Libraries; Expression of Cloned Genes; Oligonucleotides - Their Use in Gene Cloning; Site-Specific Mutagenicity; and Microdissection and Microcloning. 